\title{
Hierarchical Clustering-Based Navigation of Image Search Results
}

\author{
Haoyang Ding \\ Institute of Automation \\ Chinese Academy of Sciences \\ Beijing 100190, China \\ +86-10-62542971 \\ hyding@nlpr.ia.ac.cn
}

\author{
Jing Liu \\ Institute of Automation \\ Chinese Academy of Sciences \\ Beijing 100190, China \\ +86-10-62632267 \\ jliu@nlpr.ia.ac.cn
}

\author{
Hanqing Lu \\ Institute of Automation \\ Chinese Academy of Sciences \\ Beijing 100190, China \\ +86-10-62542971 \\ luhq@nlpr.ia.ac.cn
}

\begin{abstract}
Usually, the image search results contain multiple topics on semantic level and even semantically consistent images have diverse appearances on visual level. How to organize the results into semantically and visually consistent clusters becomes a necessary task to facilitate users' navigation. To attack this, HiCluster, an effective method to organize image search results is designed in this paper, which employs both textual and visual analysis. First, we extract some query-related key phrases to enumerate specific semantics of the given query and cluster them into some semantic clusters using K-lines-based clustering algorithm. Second, the resulting images corresponding to each key phrase are clustered with Bregman Bubble Clustering (BBC) algorithm, which partially groups images in the whole set while discarding some scattered noisy ones. At last, a novel user interface (UI) is designed to provide users with the diverse and helpful information based on the hierarchical clustering structure. Experiments on web images demonstrate the effectiveness and potential of the system.
\end{abstract}

\section{Categories and Subject Descriptors}

H.3.3 [Information storage and retrieval]: Information Search and Retrieval - clustering. H.5.3 [Information interfaces and presentation]: Group and Organization Interfaces - Web-based interaction.

\section{General Terms}

Algorithms, Design, Experimentation.

\section{Keywords}

Image search, Search result clustering, User interface

\section{INTRODUCTION}

Typically, an image search engine, such as Google or Yahoo, returns a ranked list of search results according to their relevance to a given query. However, due to the query's polysemy, the results always contain multiple topics and they are mixed together.

Permission to make digital or hard copies of all or part of this work for personal or classroom use is granted without fee provided that copies are not made or distributed for profit or commercial advantage and that copies bear this notice and the full citation on the first page. To copy otherwise, or republish, to post on servers or to redistribute to lists, requires prior specific permission and/or a fee.

MM'08, October 26-31, 2008, Vancouver, British Columbia, Canada. Copyright 2008 978-1-60558-303-7/08/10...\$5.00.

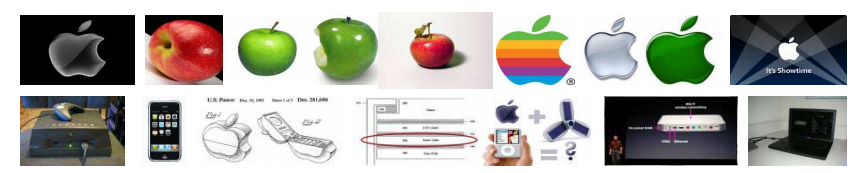

Figure 1. Top 16 returns of query "apple" in Google's image search engine.

For the example shown in Fig. 1, the images relevant to 'apple' may be 'apple computer', 'apple fruit', 'apple logo', 'apple iPod', 'apple iphone' and so on. This makes the search on the mixed list become a time-consuming and laborious task. Thus, the clustering-based navigation, which is provided with semantically and visually consistent clusters, facilitates web users to find their desired images efficiently.

Some methods for image search result clustering were proposed in literatures. In traditional Content-Based Image Retrieval (CBIR), image clustering techniques are used to present a friendly user interface [1], which helps to make more meaningful presentations of search results. However, as the images were described only with the visual features, it is hard to get consistent clusters from semantic perspective. Deng et al. [1] proposed a hierarchical clustering method using visual, textual and link analysis and finally obtained different semantic clusters. However, they could not present a semantic explanation of each cluster, and so the meaning of each cluster is difficult to understand. IGroup [2] used a clustering algorithm for web image search results. It first identifies several key phrases related to a given query, and then assigns all the resulting images to the corresponding phrases. However, the possibly consistent clusters on semantics are organized with an arbitrary order. This tends to make users puzzled because there are still many similar key phrases on semantic level. Besides, since the visual feature is not used, the coherence on visual appearance and the representative thumbnails are not available in the application.

In this paper, we design HiCluster an effective framework based on a hierarchical clustering structure in order to organize the search results convenient for users browsing. First, several queryrelated key phrases are extracted by exploring search results from general textual search engines. Then, the K-lines-based clustering algorithm is utilized to cluster the key phrases into semantic clusters. Second, the resulting images corresponding to each key phrase are grouped into some visually coherent clusters, in which Bregman Bubble Clustering (BBC) algorithm is applied to partially group images in the whole set so as to discard some scattered noisy ones. Finally, a novel UI is designed to make the best of the hierarchical clustering results. It displays the semantic 
clusters by putting the appropriate titles together and further presents the visually and semantically coherent clusters under each title by assigning some representative thumbnails. Thus, HiCluster provides users an informative resulting page with the results as diverse and relevant as possible.

The rest of this paper is organized as follows. The hierarchical clustering algorithm is introduced in Section 2. The Web image search UI is presented in Section 3. In Section 4, the experimental evaluations are reported. The paper is concluded in Section 5.

\section{HIERARCHICAL CLUSTERING}

In order to help users find the required images quickly and conveniently from the mixed resulting images list, a hierarchical clustering is used to organize the results into semantically and visually consistent clusters. The flowchart of the proposed algorithm is illustrated in Fig. 2.

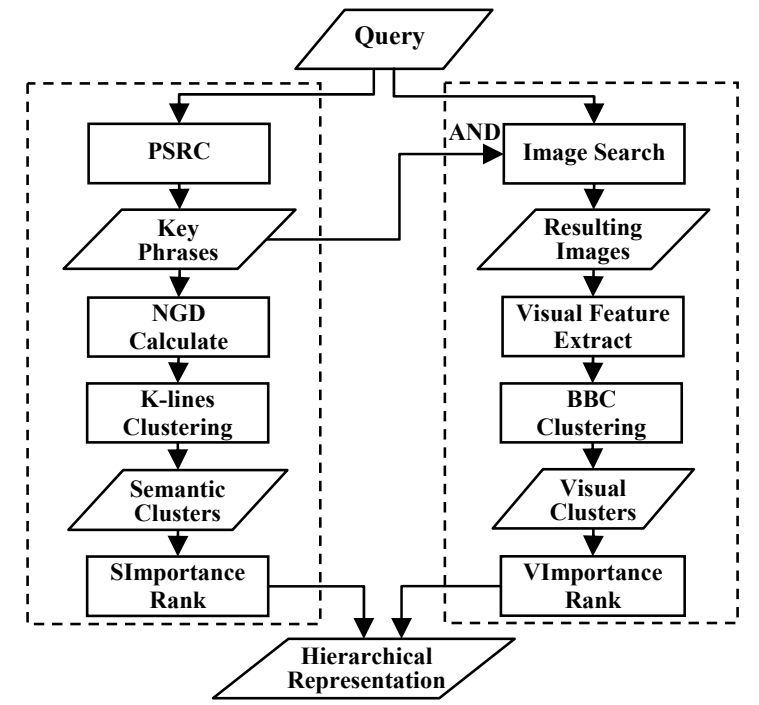

Figure 2. Flowchart of the hierarchical clustering algorithm for Web image search result.

\subsection{Semantic Clustering}

Since the semantics of a query may be diverse, it is necessary to organize the resulting images of the query according to the different semantics. In the following, we will extract some queryrelated key phrases to represent specific semantics of the query and then cluster them into different semantic clusters by exploring their semantic similarities.

At the beginning, the key phrases are created by the page search results clustering method (PSRC) [3]. Given a query and its ranked list of resulting documents (a list of titles and snippets) returned by a textual Web search engine, we first extracts all possible phrases (n-grams) from the results, and then calculates several properties for each phrase such as phrase frequencies, document frequencies, and phrase length and more. A regression learning model from training data is applied to combine these properties into a salience score. The key phrases are ranked by the salience scores, and the top-ranked phrases are taken as key phrases.

Given the key phrases relevant to a given query, the K-linesbased clustering algorithm [4] is selected to organize them into some semantically consistent clusters, due to its assumption that

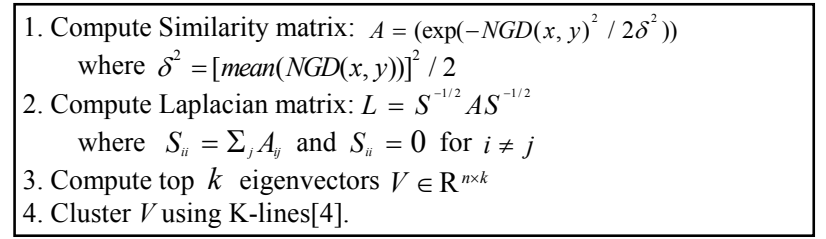

\section{Figure 3. Framework of K-lines-based clustering}

elements in each cluster are approximated by a line instead of a spherical, reinforces the block structure of the semantic affinity matrix. Based on the framework in Fig. 3, the method clusters data points by exploring the pair-wise similarities. Here, Normalized Google Distance (NGD) as Eq.1 is used to measure the semantic relevance between any phrase-pair.

$$
N G D(x, y)=\frac{\max \{\log f(x), \log f(y)\}-\log f(x, y)}{\log N-\min \{\log f(x), \log f(y)\}}
$$

where $x$ and $y$ are two query words, $G$ is the total number of web pages indexed by Google, $f(x)$ is the count of pages where word $x$ appears, and $f(x, y)$ is the count of pages where both $x$ and $y$ appear.

At last, the clusters are ranked in descending order of their semantic importance defined as:

$$
\text { SImportance }(m)=\frac{1}{N_{m}} \sum_{i=1}^{N_{m}} \operatorname{ResultSize}(i)
$$

where $N$ is the number of key phrases in the $m$ th cluster, ResultSize(i) is the resulting images number of the $i$ th key phrase.

This semantic cluster organization can not only give users a more clear representation by putting the semantic similar key phrases together, but also help them focus on more important semantics with the prior presentation of the more dominant clusters.

\subsection{Visual Clustering}

Even semantically consistent resulting images usually have diverse appearances on visual level. For instance, the resulting images of 'apple fruit' contain both 'green' and 'red' apple images. With visual clustering, the 'green' and 'red' apple images can be displayed in the different clusters so as to make users convenient to find exact results with visual consistence.

A lot of clustering algorithms have been applied to image search result clustering, such as k-means [5] and spectral clustering techniques [1]. They assume that every data point ought to be assigned to at least one cluster. However, due to the limitations of current searching techniques, only partial returned images are relevant to the query and several irrelevant images are mixed. From this view, we apply a new clustering scheme, Bregman Bubble Clustering (BBC) [6] to visually cluster the images corresponding to each key phrase. The algorithm obtains the dominant clusters by partially grouping images in the whole set while discarding some scattered noisy ones. From the statistical perspective, it is reasonable to believe that the dominant clusters as main visual representations can reflect the semantics of the given key phrase more efficiently.

Here, we present a fast iterative solution for $\mathrm{BBC}$, which guarantees a local minimum. Given $k$ centers from $n$ data points and a desired threshold $s$ of each cluster size, there are three steps: (1) assigning each point to the nearest center, (2) picking points closest to their centers first until s points are picked, and (3) updating the centers. These three steps are repeated until there is no change in assignment between two successive iterations. To address BBC's sensitivity to the initial seeds, a process called 
Pressurization [6] is introduced. Specially, a parameter set of $\left\{s_{j}\right\}$, where $s_{j}=s+\left[(n-s) \cdot r^{j-1}\right]$ is used instead of $s$ in $j$ th iteration. And $r \in(0,1)$ controls the increasing rate of pressure.

After the visual clustering is finished, the images closest to the cluster center are chosen as its representative images, and all the images of the cluster are sorted in descending order of their distance from the cluster center. Different visual clusters are ranked in descending order of their importance defined as:

$$
\operatorname{VImportance}(n)=\operatorname{ClusterSize}(n) / \sigma_{n}
$$

where ClusterSize is the number of images in the $n$th visual cluster, $\sigma_{n}$ is the standard deviation of the cluster. From the measurement, the large and dense clusters are preferred.

The visual clustering organization presents users a more informative and convenient navigation by putting the visual similar images together, and tagging them with a representative thumbnail. Besides, the ranked presentation of several dominant image clusters is facilitated to lead users' attention to the images which can reflect the semantics of the key phrase efficiently.

\section{USER INTERFACE}

In order to make the best use of the hierarchical clustering result, a novel UI is designed as shown in Fig.4. It is composed of three parts: query input view (QView), hierarchical cluster navigation view (HCView) and result list view (RView).

\subsection{Query Input View}

The query input view is placed in the top frame as in Fig.4. Users can input query in the edit box. And also, they can use sophisticated queries, such as 'dog AND cat' or 'dog OR cat' or 'dog AND NOT cat'.

\subsection{Hierarchical Cluster Navigation View}

The hierarchical cluster information is organized in the hierarchical cluster navigation view which is embedded in the left frame. The key phrases with similar semantics are put together and bounded with an orange rectangle to represent a semantic cluster. The semantic clusters are in descending order of their importance. Under every key phrase, there are representative image thumbnails for the visual clusters of the resulting images. The visual clusters are also in descending order of the measurement on its dominance. When the key phrase or the

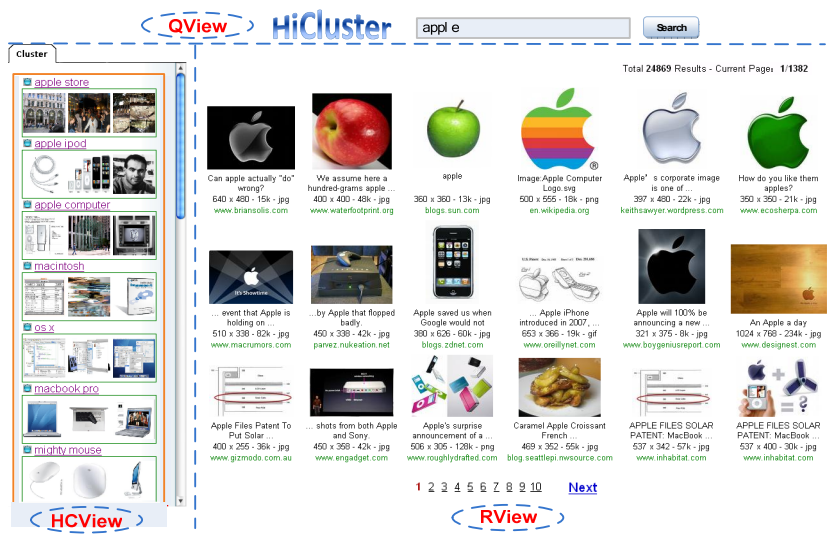

Figure 4. Snapshot of UI for HiCluster representative image is clicked, the results will be displayed in result list view which will be discussed in next sub-section.

\subsection{Result List View}

The result list view residing in the main frame of the page has three states. The first state as shown in Fig. 4 displays a ranked list of resulting images, which is similar to existing image search engine. In the view, there are a pre-defined number of image thumbnails with related information, such as image name, image size and Web site containing the target image. From Fig.5, it can be seen that the second state shows each visual cluster with four representative images. It appears when the cluster name in the hierarchical cluster navigation view is clicked. The third state displays the resulting images included in the corresponding visual cluster when the representative image of the visual cluster under the cluster name is clicked.

\section{EXPERIMENTS}

All the data used in our experiments are crawled by searching on Google and Flickr. We select 1000 popular keywords as the queries. For each query, 1000 top-ranked images are crawled and their corresponding web pages are also downloaded. With an HTML parser which depends on DOM-tree structure, the textual information of each image, which includes the words in title, URL, ALT tag, anchor text and surrounding text, is extracted for the image indexing on semantic level. Additionally, a 168dimensional visual feature vector for each image is extracted. It includes 144-dimensional color correlogram and 24-Dimensional Polynomial Wavelet Tree (PWT).

To evaluate the performance of HiCluster, we randomly select 14 keywords to perform the hierarchical clustering. 12 participants are involved, and three searching systems of HiCluster, IGroup [2], and Google are compared together in the following experiments. At first, we designed a user study for UI satisfaction evaluation on the whole. And then, the search effort is used to evaluate the consumption of physical or mental energy to accomplish search. At last, the performance efficiency in search is analyzed to prove the effectiveness of HiCluster.

\subsection{UI Satisfaction Evaluation}

In UI satisfaction evaluation, the participants are asked to evaluate all three UIs with arbitrary queries they liked, while the UIs are presented to the participants in a random order. The three UIs are ranked with "=", ">"or" $>>$ ", where "=" means no

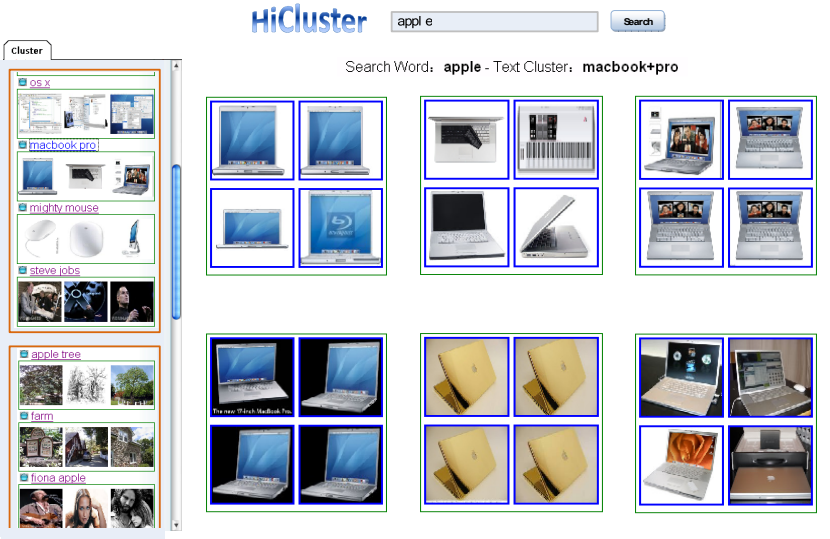

Figure 5. Visual clusters navigation UI 


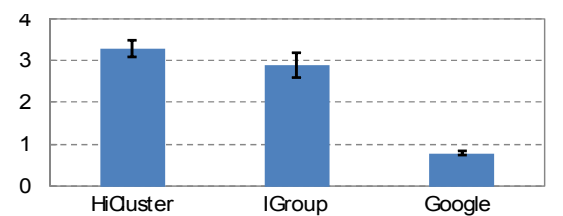

Figure 6. Mean Ratings of three UIs

difference, ">" means better and " $>>$ " means much better. In order to quantize the rank results, the ranking pair is converted into the rating. The worst one is assigned with the number 1 . If $X=Y$, the rating of $X$ will be the rating of $Y$; if $X>Y$, the rating of $X$ will be the rating of $Y$ plus 1 ; if $X>>Y$, the rating of $X$ will be the rating of $\mathrm{Y}$ plus 2. The Figure 6 illustrates that HiCluster $>$ IGroup $>$ Google. The ANOVA test is executed. The result is $\mathrm{F}(2,35)=50.1, \mathrm{p}<0.05$ which means that the difference of the three UIs is very significant. Furthermore, $F(11,35)=1.80, p>0.05$ which indicate that the difference between participants is not very significant.

\subsection{Search Effort Evaluation}

In the evaluation, the participants are required to use all three UIs with queries within a specified period. Here, the search effort includes the formation and input of a query, the clicking of page links, and the expense on IGroup clusters, HiCluster semantic clusters, or visual clusters. Then the search efforts for Google, IGroup and HiCluster respectively are defined as:

$$
\begin{gathered}
\mathrm{SE}_{\text {Google }}=\mathrm{N}_{\text {query }}+\mathrm{N}_{\text {page links clicked }} \\
\mathrm{SE}_{\text {IGroup }}=\mathrm{N}_{\text {query }}+\mathrm{N}_{\text {page links clicked }}+\mathrm{N}_{\text {cluster name clicked }} \\
\mathrm{SE}_{\text {HiCluster }}=\mathrm{N}_{\text {query }}+\mathrm{N}_{\text {page links clicked }}+\mathrm{N}_{\text {semantic cluster clicked }}+\mathrm{N}_{\text {visual cluster clicked }}
\end{gathered}
$$

where $\mathrm{N}$ stands for the number of the items in the footnote.

Figure 7 illustrate the search effort comparison among them corresponding to different semantic queries. Obviously, the participants spent less search effort with HiCluster than with IGroup or Google. It means that HiCluster makes search process more convenient and easier for users. Specially, the queries with polysemy such as 'apple' and 'tiger' save more search effort than the queries with specific semantics such as 'cat' and 'dog'. This indicates that the HiCluster can effectively reduce the difficulty of the polysemous query search.

\subsection{Search Performance Evaluation}

In order to evaluate the search performance, the number of retrieved images, the visual cluster coverage and the semantic cluster coverage of the retrieved images are analyzed. Figure 8 presents the comparison among the three systems.

From Figure 8, we can see: Firstly, the number of images

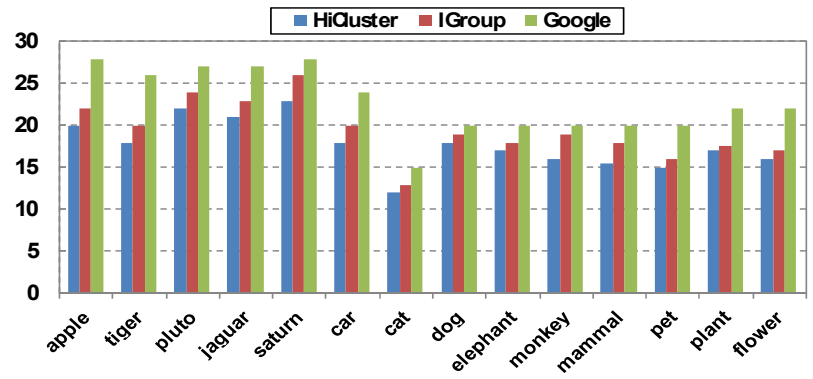

Figure 7. Search effort Comparison

retrieved by HiCluster $(\mathrm{M}=17.2, \mathrm{SD}=2.1)$ is larger than that of

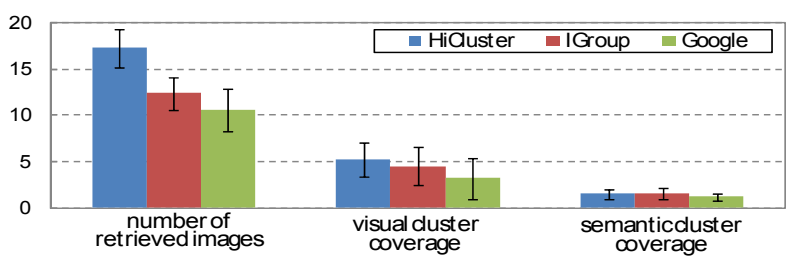

Figure 8. Search performance comparison

IGroup $(\mathrm{M}=12.3, \mathrm{SD}=1.8)$ and Google $(\mathrm{M}=10.6, \mathrm{SD}=2.3)$. It demonstrates that the hierarchical clustering structure in HiCluster facilitate the access to more relevant images. Secondly, the visual cluster coverage of the images retrieved by HiCluster $(\mathrm{M}=5.2$, $\mathrm{SD}=1.9)$ is close to that of IGroup $(\mathrm{M}=4.5, \mathrm{SD}=2.1)$, but larger than that of Google $(\mathrm{M}=3.2, \mathrm{SD}=2.2)$. The wider coverage proves that the visual clustering method provide users with more diverse images relevant to search goals. Thirdly, the semantic cluster coverage of the retrieved images from any of the three systems is small, i.e., the semantic meanings of the retrieved images are concentrated. It is reasonable to think that users usually have relatively clear search intentions before submitting a query. Then, the small semantic cluster coverage fits the fact very well and proves the effectiveness of our semantic clustering method.

\section{CONCLUSION}

In this paper, HiCluster, an effective framework for the hierarchical clustering-based navigation of image search results is described. The method has the following unique advances. First, the K-lines-based semantic clustering organization, which is performed by putting the semantic similar key phrases together, provides users a concise understanding of the query semantic meanings. Second, the BBCbased visual clustering for the images in each semantic sub-cluster presents users a more informative and convenient navigation by putting the visual similar images together, and tagging them with a representative thumbnail. Third, a friendly UI is designed to make the best of the hierarchical clustering solution. At last, the reasonable and comprehensive evaluations are performed to demonstrate the effectiveness of the framework.

\section{ACKNOWLEDGEMENTS}

The research was supported by National 863 Project (2006AA01Z315), National Natural Science Foundation of China (60675003), and Beijing Natural Science Foundation (4072025).

\section{REFERENCE}

[1] D. Cai, X. He, Z. L, W.Y. Ma, J.R. Wen. Hierarchical Clustering of www Image Search Results Using Visual, Textual and Link Information. ACM Multimedia 2006, pp: 952 - 959.

[2] F. Jing, C. Wang, Y. Yao, K. Deng, L. Zhang, W.Y. Ma. IGroup: Web Image Search Results Clustering. Proc. ACM Multimedia 2006.

[3] H.J Zeng, Q.C He, Z. Chen, W.Y. Ma, J. Ma. Learning to Cluster Web Search Results. ACM SIGIR'04, pp: 210-217.

[4] Igor Fischer. New Method for Spectral Clustering. Technical Report No. IDSIA-12-04, Hebrew University, Israel,2004.

[5] T. Deselaers, D. Keysers, and H. Ney, Clustering visually similar images to improve image search engines, in I nformatiktage 2003 der Gesellschaft fur Informatik, 2003.

[6] A. Banerjee, I. S. Dhillon, and J. Ghosh. Clustering with bregman divergences. JMLR, vol. 6, pp. 1705- 1749, 2005. 